\title{
On the Performance Evaluation of TH and DS UWB MIMO Systems
}

\author{
Wipawee Siriwongpairat, Masoud Olfat, and K. J. Ray Liu \\ Department of Electrical and Computer Engineering, University of Maryland, College Park, MD 20742.
}

\begin{abstract}
In this paper, we analyze the performance of UltraWideBand (UWB) Multiple-Input Multiple-Output (MIMO) systems employing various modulation and multiple access schemes, including Time Hopping (TH) $M$-ary Pulse Position Modulation (MPPM), TH Binary Phase Shift Keying (BPSK), and Direct Sequence (DS) BPSK. We quantify the performance merits of UWB Space-Time (ST) systems regardless of the specific coding scheme. For each modulation scheme, we introduce a framework that enables us to compare UWB MIMO systems with conventional UWB singleantenna systems in terms of diversity and coding gain. Moreover, we adopt the Real Orthogonal Design (ROD) as the engine code for UWB ST codes. We find the closed form expressions of the average Pairwise Error Probability (PEP) bound for all of the schemes under the hypothesis of Nakagami flat fading channels. The extension to frequency-selective fading case is also briefly addressed. Finally, simulation results are presented to support the theoretical analysis.
\end{abstract}

\section{INTRODUCTION}

Ultra-WideBand (UWB) technology is defined as a transmission scheme that occupies a bandwidth of more than $20 \%$ of its center frequency, or typically more than $500 \mathrm{M} \mathrm{Hz}$. UWB signal is characterized by a train of extremely short duration pulses, where the information is carried in the shifted position, polarity, or amplitude of the pulses [1]. The Multiple-Access (MA) capability of UWB system is attained by incorporating the UWB signal with a pseudo-random Time Hopping ( $\mathrm{TH})$ or spreading sequence [2]. With its unique properties of MA ability, multipath resistance, low power requirement and low implementation cost, UWB system is a viable candidate for short range communications in dense multipath environments, especially indoor wireless and home entertainment systems.

Multiple-Input Multiple-Output (MIMO) system is well known for its potential of improving system performance under multipath scenarios. In particular, a large number of propagation paths between transmit and receive antennas can be utilized to combat channel fading effect and gain link reliability. It has been shown that by employing multiple transmit and receive antennas, both space and time diversities can be achieved, and hence system performance can be significantly improved. In order to approach such improvements, several Space-Time (ST) coding schemes have been proposed, for example in [3], [4].

To exploit the advantages of both UWB and MIMO systems, UWB ST coded scheme has been proposed [5]. The authors in [5] suggested a TH $M$-ary Pulse Position Modulation (MPPM) ST coded system based on repetition codes which is a special case (reduced rate) of what we present in this work. In this paper, we consider UWB ST systems employing different modulation and MA techniques, including TH MPPM [2], TH Binary Phase Shift Keying (BPSK) [1], and Direct Sequence (DS) BPSK [6]. The performance comparisons of TH and DS schemes for single antenna systems have been studied in [7], [8]. Here, we quantify the performance figures of UWB ST systems regardless of the specific coding scheme. We first assume that channels are flat over a few time slots to be able to devise a theoretical framework to characterize the performances of UWB ST systems with the diversity and the coding advantages. Then, generalization to the more realistic case of frequency-selective channels is briefly discussed. Even though the antenna characteristics does not remain constant over the large bandwidth of UWB systems, by applying the second derivative of Gaussian pulses at the receiver, we can equalize the variations of antenna characteristics due to large frequency bandwidth [9]. We also utilize the Real Orthogonal Design (ROD) [4] as the engine code for UWB ST codes. Our simulation results show that for every modulation scheme, the performance of UWB MIMO system is remarkably improved compared to that of the conventional UWB Single-Input Single Output (SISO) system.

The rest of the paper is organized as follows. In Section II, we introduce the transmitted ST signals for TH and DS MIMO systems. The receiver structures and the analysis of received signals are presented in Section III. In Section IV, we investigate the system performances in terms of Pairwise Error Probability (PEP). The performances of UWB ROD ST codes with different rates are evaluated in Section V. The generalization to frequencyselective case is summarized in Section VI. Section VII describes numerical results and finally Section VIII concludes the paper.

\section{System Models}

We consider UWB MIMO multiuser environment with $N_{u}$ users, each equipped with $N_{t}$ transmit antennas, and a receiver with $N_{r}$ receive antennas. At each transmitter, the input bit stream (coded or uncoded) is divided into segments of $b$ bits, forming a sequence of $M=2^{b}$-ary symbols. The symbol sequence is further divided into blocks of $N_{b}$ symbols. Each block is encoded into a ST codeword to be transmitted over $N_{t}$ transmit antennas during $K$ time slots. Since $K$ time slots are required to transmit $N_{b}$ symbols, the code rate is $R=N_{b} / K$. Each ST codeword matrix can then be expressed as a $K \times N_{t}$ matrix $D_{u}$ whose $(k, i)^{t h}$ element is $d_{u}^{i}(k)$, which represents the $M$-ary symbol transmitted by the $u^{t h}$ user at transmit antenna $i$ over time slot $k$. The transmitter converts the $i^{t h}$ column of the ST codeword matrix into UWB signal which is then transmitted from transmit antenna $i$. The resultant ST UWB signal can be expressed as a $K \times N_{t}$ matrix $\tilde{\mathbf{X}}_{u}(t)$ whose $(k, i)^{t h}$ element is the transmitted UWB signal $\tilde{x}_{u}^{i}(k ; t)$ corresponding to the symbol $d_{u}^{i}(k)$. The signal $\tilde{x}_{u}^{i}(k ; t)$ depends on the particular MA and modulation schemes and will be discussed in the following subsections. 


\section{A. TH-MPPM}

UWB signal comprises short length pulses with durations generally in the order of nanoseconds $(n s)$. The conventional UWB modulation scheme is based on TH-MPPM in which the data symbols are carried in the fine time shift of the pulses. The $u^{\text {th }}$ user's transmitted waveform at the $i^{\text {th }}$ transmit antenna can be described as [2]:

$$
\tilde{x}_{u}^{i}(t)=\sum_{k=0}^{K-1} \sqrt{\frac{E_{u}}{N_{t}}} \tilde{w}\left(t-k T_{f}-c_{u}(k) T_{c}-T_{d_{u}^{i}(k)}\right),
$$

where $\tilde{w}(t)$ is the transmitted monocycle of duration $T_{w}$, and $T_{f}$ is the pulse repetition period with $T_{f} \gg T_{w}$. The monocycle is normalized to have unit energy, and the factor $\sqrt{E_{u} / N_{t}}$ ensures that the total transmitted energy of the $u^{t h}$ user is $E_{u}$ during each frame interval, independent of the number of transmit antennas. Each frame contains $N_{c}$ subinterval of $T_{c}$ seconds where $N_{c} T_{c} \leq T_{f}$. The TH sequence of the $u^{t h}$ user is denoted by $\left\{c_{u}(k)\right\}, 0 \leq c_{u}(k) \leq N_{c}-1$. It provides an additional time shift of $c_{u}(k) T_{c}$ seconds to the $k^{\text {th }}$ monocycle in order to allow MA without catastrophic collisions. We denote the monocycle time shift corresponding to the $u^{\text {th }}$ user's transmitted symbol at transmit antenna $i$ over frame $k$ as $T_{d_{u}^{i}(k)} \in\left\{0=T_{0}<T_{1}<\right.$ $\left.\cdots<T_{M-1}\right\}$. These modulation delays are chosen to satisfy $T_{m}-T_{m-1} \geq T_{w}$ to make the $M$ possible pulses orthogonal at the receiver, resulting in an $M$-ary orthogonal signaling scheme. Thus, the hop duration satisfies $T_{c} \geq M T_{w}$.

The UWB signal in (1) can also be expressed as [5], [10]:

$$
\tilde{x}_{u}^{i}(t)=\sum_{m=0}^{M-1} \sqrt{\frac{E_{u}}{N_{t}}} \sum_{k=0}^{K-1} s_{d_{u}, m}^{i}(k) \tilde{w}_{u, m}^{k}(t),
$$

where $\tilde{w}_{u, m}^{k}(t) \triangleq \tilde{w}\left(t-k T_{f}-c_{u}(k) T_{c}-T_{m}\right), s_{d_{u}, m}^{i}(k) \triangleq$ $\delta\left(d_{u}^{i}(k)-m\right)$, for $m=0,1, \ldots, M-1$ and $\delta(\cdot)$ denotes the Dirac delta function. In (2), the UWB signal is decomposed into $M$ waveforms, each comprising the monocycles with modulation delay of $T_{m}$ seconds. During the $k^{t h}$ frame, only the $m^{\prime t h}$ decomposed waveform corresponding to the transmitted symbol $d_{u}^{i}(k)=m^{\prime}$ contains the monocycle. An example of TH Binary PPM (BPPM) signal and its decomposition is depicted in Fig. 1.

\section{B. $T H-B P S K$}

TH-BPSK scheme exploits the TH sequence concept as does in the TH-MPPM scheme. However, the information in TH-BPSK system is carried in the polarity rather than time delay of the pulses. The transmitted UWB TH-BPSK signal is given by [1]:

$$
\tilde{x}_{u}^{i}(t)=\sum_{k=0}^{K-1} \sqrt{\frac{E_{u}}{N_{t}}} d_{u}^{i}(k) \tilde{w}\left(t-k T_{f}-c_{u}(k) T_{c}\right),
$$

where $d_{u}^{i}(k)$ represents the transmitted binary symbol, $d_{u}^{i}(k) \in$ $\{-1,1\}$. Similar to the TH-MPPM case, each frame contains only one monocycle with a delay corresponding to the assigned $\mathrm{TH}$ sequence, $\left\{c_{u}(k)\right\}, 0 \leq c_{u}(k) \leq N_{c}-1$. The hop duration is chosen such that $T_{c} \geq T_{w}$ and $N_{c} T_{c} \leq T_{f}$. The monocycle is normalized to have unit energy, and the total transmitted energy per frame of the $u^{t h}$ user is $E_{u}$.

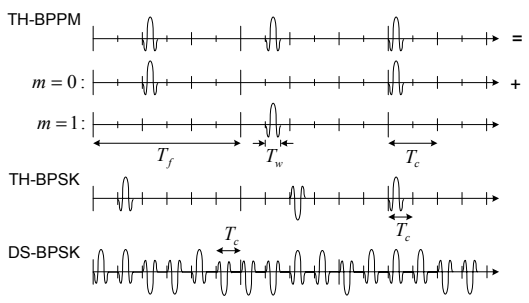

Fig. 1: UWB signals with various modulation and MA techniques

\section{DS-BPSK}

In DS-BPSK system, the binary symbol $d_{u}^{i}(k) \in\{-1,1\}$ to be transmitted over the $k^{\text {th }}$ frame interval is spread by a sequence of multiple monocycles $\left\{c_{u}(l) \tilde{w}\left(t-k T_{f}-l T_{c}\right)\right\}_{l=0}^{N_{c}-1}$ whose polarities are determined by the spreading sequence $\left\{c_{u}(l)\right\}_{l=0}^{N_{c}-1}$, $c_{u}(l) \in\{-1,1\}$. The transmitted signal can be described as [6]:

$$
\tilde{x}_{u}^{i}(t)=\sum_{k=0}^{K-1} \sqrt{\frac{E_{u}}{N_{t} N_{c}}} d_{u}^{i}(k) \sum_{l=0}^{N_{c}-1} c_{u}(l) \tilde{w}\left(t-k T_{f}-l T_{c}\right) .
$$

The frame interval $T_{f}$ is divided into $N_{c}$ segments of $T_{c}$ seconds. Here, the hop period is chosen to satisfy $T_{c} \geq T_{w}$, which results in the orthogonality between the monocycles contained in a sequence regardless of the particular spreading code. Since each frame contains $N_{c}$ normalized monocycles, we introduce the factor $\sqrt{1 / N_{c}}$ to ensure that the sequence of $N_{c}$ monocycles has unit energy. With the monocycle sequence being normalized and the factor $\sqrt{E_{u} / N_{t}}$ being included, the $u^{t h}$ user's transmitted energy per frame is $E_{u}$.

Fig. 1 illustrates an example of UWB signals employing THBPPM, TH-BPSK and DS-BPSK schemes.

\section{RECEIVER DESCRIPTIONS}

We have simplified the channel model by considering real, mutually independent, flat fading channels over a block of $K$ time slots. The channel fading coefficient from the $i^{\text {th }}$ transmit antenna of the $u^{t h}$ user to the $j^{t h}$ receive antenna is denoted by $h_{u}^{i j}$. The amplitudes of $h_{u}^{i j}$ are assumed to be Nakagami- $\tilde{m}$ distributed with the average power $\Omega_{u}$. We assume that the Channel State Information (CSI) is known at the receiver but not at the transmitter. At the receive antenna output, the shape of transmitted monocycle $\tilde{w}(t)$ is transformed to its second derivative due to the effect of propagation channel and the variation of antenna characteristics caused by large bandwidth [9]. Denoting the received monocycle as $w(t)$ and defining $x_{u}^{i}(t)$ similar to the transmitted waveform $\tilde{x}_{u}^{i}(t)$ by replacing $\tilde{w}(t)$ with $w(t)$, the received signal at receive antenna $j$ can be modelled as:

$$
r^{j}(t)=\sum_{u=0}^{N_{u}-1} \sum_{i=0}^{N_{t}-1} h_{u}^{i j} x_{u}^{i}\left(t-\tau_{u}\right)+n^{j}(t),
$$

where $n^{j}(t)$ denotes real additive white Gaussian noise process with zero mean and two-sided power spectral density $N_{0} / 2$, and $\tau_{u}$ represents asynchronous delay associated to the $u^{t h}$ user. Considering the first user as the desired user, the received signal model in (5) can be restated as:

$$
r^{j}(t)=\sum_{i=0}^{N_{t}-1} h_{0}^{i j} x_{0}^{i}\left(t-\tau_{0}\right)+n_{M U}^{j}(t)+n^{j}(t),
$$




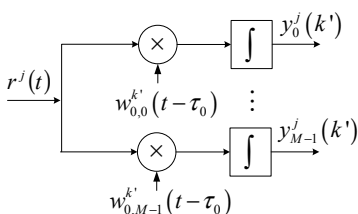

Fig. 2: A bank of correlators at receive antenna $j$ in TH-MPPM system.

where $n_{M U}^{j}(t)=\sum_{u=1}^{N_{u}-1} \sum_{i=0}^{N_{t}-1} h_{u}^{i j} x_{u}^{i}\left(t-\tau_{u}\right)$ is the signal received from other users. For the signal transmitted from the desired user, we assume that the receiver has perfect synchronization and the knowledge of the $\mathrm{TH}$ and spreading sequence, i.e., with the first user being the desired user, $\tau_{0}$ and $\left\{c_{0}(k)\right\}$ are assumed known at the receiver. We also assume that the receiver knows the received monocycle $w(t)$. The receiver comprises a bank of correlators, each adopting the delayed versions of $w(t)$ as the reference pulses, and a Maximum Likelihood (ML) decoder where the decoding process is performed jointly across all $N_{r}$ receive antennas.

\section{A. $T H-M P P M$}

In order to estimate the transmitted $M$-ary symbol sequence, the received signal at the $j^{\text {th }}$ receive antenna is correlated with the locally generated signals $\left\{w_{0, m^{\prime}}^{k^{\prime}}\left(t-\tau_{0}\right)\right\}_{m^{\prime}=0}^{M-1}$ as shown in Fig. 2. The output of the $m^{\prime t h}$ correlator can be calculated as:

$$
\begin{aligned}
y_{m^{\prime}}^{j}\left(k^{\prime}\right) & =\int_{-\infty}^{\infty} w_{0, m^{\prime}}^{k^{\prime}}\left(t-\tau_{0}\right) r^{j}(t) d t \\
& \triangleq y_{d, m^{\prime}}^{j}\left(k^{\prime}\right)+n_{M U, m^{\prime}}^{j}\left(k^{\prime}\right)+n_{m^{\prime}}^{j}\left(k^{\prime}\right),
\end{aligned}
$$

where $y_{d, m^{\prime}}^{j}\left(k^{\prime}\right), n_{M U, m^{\prime}}^{j}\left(k^{\prime}\right)$, and $n_{m^{\prime}}^{j}\left(k^{\prime}\right)$ denote the correlator output corresponding to the desired transmitted information, MA interference and thermal noise, respectively. Using (2), $y_{d, m^{\prime}}^{j}\left(k^{\prime}\right)$ is simplified to:

$$
y_{d, m^{\prime}}^{j}\left(k^{\prime}\right)=\sqrt{\frac{E_{0}}{N_{t}}} \sum_{i=0}^{N_{t}-1} h_{0}^{i j} s_{d_{0}, m^{\prime}}^{i}\left(k^{\prime}\right),
$$

where the interference caused by the pulse correlation is neglected with the assumption that $\left|T_{m^{\prime}}-T_{m}\right| \geq T_{w}$ for $m \neq m^{\prime}$. Now, the correlator output $y_{m^{\prime}}^{j}\left(k^{\prime}\right)$ can be written as:

$$
y_{m^{\prime}}^{j}\left(k^{\prime}\right)=\sqrt{\frac{E_{0}}{N_{t}}} \sum_{i=0}^{N_{t}-1} h_{0}^{i j} s_{d_{0}, m^{\prime}}^{i}\left(k^{\prime}\right)+n_{t o t, m^{\prime}}^{j}\left(k^{\prime}\right),
$$

in which $n_{t o t, m^{\prime}}^{j}\left(k^{\prime}\right)=n_{M U, m^{\prime}}^{j}\left(k^{\prime}\right)+n_{m^{\prime}}^{j}\left(k^{\prime}\right)$ represents MA interference plus thermal noise. Rewriting the correlator outputs of all $N_{r}$ receive antennas in the vector form, we obtain

$$
\mathbf{Y}=\sqrt{\frac{E_{0}}{N_{t}}} \mathbf{S}_{D_{0}} \mathbf{H}_{0}+\mathbf{N}_{t o t}
$$

where $\mathbf{S}_{D}$ is an $M K \times N_{t}$ matrix constructed from the $\mathrm{ST}$ symbol $D$ as follows:

$$
\mathbf{S}_{D}=\left[\begin{array}{llll}
S_{D}^{T}(0) & S_{D}^{T}(1) & \cdots & S_{D}^{T}(K-1)
\end{array}\right]^{T},
$$

in which $S_{D}(k)=\left[\begin{array}{llll}\mathbf{s}_{d}^{1}(k) & \mathbf{s}_{d}^{2}(k) & \cdots & \mathbf{s}_{d}^{N_{t}}(k)\end{array}\right]$ is composed of $N_{t}$ vectors. Each $\mathbf{s}_{d}^{i}(k)$ is an $M \times 1$ vector $\mathbf{s}_{d}^{i}(k)=$ $\left[s_{d, 0}^{i}(k), s_{d, 1}^{i}(k), \cdots, s_{d, M-1}^{i}(k)\right]^{T}$ where $s_{d, m}^{i}(k)=\delta\left(d^{i}(k)-\right.$ $m)$ as defined previously. The channel matrix $\mathbf{H}_{0}$ is of size $N_{t} \times$
$N_{r}$ whose $(i, j)^{t h}$ element is $h_{0}^{i j}$. The correlator output matrix $\mathbf{Y}$ of size $M K \times N_{r}$ is given by $\mathbf{Y}=\left[Y^{T}(0) Y^{T}(1) \cdots Y^{T}(K-\right.$ $1)]^{T}$, where $Y^{T}(k)$ is an $M \times N_{r}$ matrix in which $(m, j)^{t h}$ entry is $y_{m}^{j}(k)$. The noise and interference matrix $\mathbf{N}_{t o t}$ has a form similar to $\mathbf{Y}$ by replacing $y_{m}^{j}(k)$ with $n_{m}^{j}(k)$. Given the CSI on MIMO channels, the decoder performs ML decoding by selecting a codeword $\hat{D}_{0}$ which minimizes the square Euclidean distance between the hypothesized and actual correlator output matrices. The decision rule can be stated as:

$$
\hat{D}_{0}=\arg \min _{D_{0}}\left\|\mathbf{Y}-\sqrt{\frac{E_{0}}{N_{t}}} \mathbf{S}_{D_{0}} \mathbf{H}_{0}\right\|^{2},
$$

where $\|\mathbf{X}\|$ denotes the Frobenius norm of $\mathbf{X}$.

\section{B. $T H / D S-B P S K$}

In BPSK system, the signal received at each receive antenna is correlated with the reference signal $v_{0}^{k^{\prime}}\left(t-\tau_{0}\right)$, where $v_{u}^{k}(t)$ stands for $w\left(t-k T_{f}-c_{u}(k) T_{c}\right)$ in case of TH-BPSK and $\sqrt{1 / N_{c}} \sum_{l=0}^{N_{c}-1} c_{u}(l) w\left(t-k T_{f}-l T_{c}\right)$ for DS-BPSK scheme. The correlator output at received antenna $j$ is given by:

$$
\begin{aligned}
y^{j}\left(k^{\prime}\right) & =\int_{-\infty}^{\infty} v_{0}^{k^{\prime}}\left(t-\tau_{0}\right) r^{j}(t) d t \\
& \triangleq y_{d}^{j}\left(k^{\prime}\right)+\underbrace{n_{M U}^{j}\left(k^{\prime}\right)+n^{j}\left(k^{\prime}\right)}_{n_{\text {tot }}^{j}\left(k^{\prime}\right)},
\end{aligned}
$$

where similar to the TH-MPPM case, $y_{d}^{j}\left(k^{\prime}\right), n_{M U}^{j}\left(k^{\prime}\right)$, and $n^{j}\left(k^{\prime}\right)$ come from the desired user signal, MA interference and thermal noise, respectively. Substituting (3) or (4) into (13), $y_{d}^{j}\left(k^{\prime}\right)$ can be shown to be

$$
y_{d}^{j}\left(k^{\prime}\right)=\sqrt{\frac{E_{0}}{N_{t}}} \sum_{i=0}^{N_{t}-1} h_{0}^{i j} d_{0}^{i}\left(k^{\prime}\right) .
$$

By combining (13) and (14) and rewriting the outputs from all $N_{r}$ correlators in the vector form, we have

$$
\mathbf{Y}=\sqrt{\frac{E_{0}}{N_{t}}} D_{0} \mathbf{H}_{0}+\mathbf{N}_{t o t},
$$

where $D_{0}$ is the desired user's transmitted ST symbol defined previously. Both matrices $\mathbf{Y}$ and $\mathbf{N}_{t o t}$ are of size $K \times N_{r}$ whose $(k, j)^{t h}$ elements are $y^{j}(k)$ and $n_{\text {tot }}^{j}(k)$, respectively. The decision rule for the ML decoder can be stated similar to (12) as:

$$
\hat{D}_{0}=\arg \min _{D_{0}}\left\|\mathbf{Y}-\sqrt{\frac{E_{0}}{N_{t}}} D_{0} \mathbf{H}_{0}\right\|^{2} .
$$

\section{Performance Analysis}

\section{A. Noise and Interference Distributions}

To analyze the performances of UWB ST systems, we first calculate the noise and MA interference statistics. From (7) and (13), $n_{m}^{j}(k)$ and $n^{j}(k)$ can be shown to be Gaussian distributed with zero mean and variance $N_{0} / 2$. To investigate the distribution of MA interference for MPPM, $n_{M U, m}^{j}(k)$, from (7) we have

$$
n_{M U, m}^{j}(k)=\sum_{u=1}^{N_{u}-1} \sum_{i=0}^{N_{t}-1} h_{u}^{i j} n_{u, m}^{i}(k),
$$


where $n_{u, m}^{i}(k) \triangleq \int_{-\infty}^{\infty} w_{0, m}^{k}\left(t-\tau_{0}\right) x_{u}^{i}\left(t-\tau_{u}\right) d t$. It has been shown in [9] that $n_{u, m}^{i}(k)$ can be approximated by Gaussian random variable with zero mean and variance $\left(E_{u} / N_{t}\right) \sigma_{a}^{2}$ where

$$
\sigma_{a}^{2} \triangleq \frac{1}{T_{f}} \int_{-\infty}^{\infty}\left[\int_{-\infty}^{\infty} w(t-s) w(t) d t\right]^{2} d s .
$$

Assuming Nakagami- $\tilde{m}$ flat fading channel coefficients, independent of the transmitted signals, and using central limit theorem, we can show that for sufficiently large $N_{t}$ and $N_{u}, n_{M U, m}^{j}(k)$ is approximately Gaussian random variable with zero mean and variance $\sigma_{a}^{2} \sum_{u=1}^{N_{u}-1} \Omega_{u} E_{u}$. Hence, the total noise and interference $n_{t o t, m}^{j}(k)=n_{M U, m}^{j}(k)+n_{m}^{j}(k)$ is zero mean Gaussian random variable with variance $\sigma_{n_{\text {tot }}}^{2}=\sigma_{a}^{2} \sum_{u=1}^{N_{u}-1} \Omega_{u} E_{u}+N_{0} / 2$. Following similar calculation steps, we can show that for BPSK system, $n_{\text {tot }}^{j}(k)$ is also approximately Gaussian distributed with zero mean and variance $\sigma_{n_{t o t}}^{2}[7]$.

\section{B. PEP Evaluation}

Since thermal noise and MA interference in the analyzed systems can be approximated with Gaussian distribution, the PEP of these systems can be calculated similarly, and the calculation is similar to the conventional narrowband MIMO system. Suppose that $D_{0}$ and $\hat{D}_{0}$ are two different transmitted ST codewords, then the PEP conditioned on the channel matrix $\mathbf{H}_{0}$ is given by [11]:

$$
P\left(D_{0} \rightarrow \hat{D}_{0} \mid \mathbf{H}_{0}\right)=\mathrm{Q}\left(\sqrt{\frac{\rho}{2 N_{t}}}\left\|\boldsymbol{\Delta}_{D_{0}} \mathbf{H}_{0}\right\|\right),
$$

where $\boldsymbol{\Delta}_{D_{0}}$ is defined as $\mathbf{S}_{D_{0}}-\mathbf{S}_{\hat{D}_{0}}$ for MPPM and $D_{0}-\hat{D}_{0}$ for BPSK systems, $\mathrm{Q}(x)$ is the Gaussian error function and

$$
\rho=\frac{E_{0}}{2 \sigma_{n_{\text {tot }}}^{2}}=\left(2 \sigma_{a}^{2} \sum_{u=1}^{N_{u}-1} \Omega_{u} \frac{E_{u}}{E_{0}}+\frac{N_{0}}{E_{0}}\right)^{-1} .
$$

For Nakagami- $\tilde{m}$ fading channels, the upper bound of the PEP can be obtained by averaging (19) over all possible channel realizations. The resultant PEP can be found in a similar fashion as in [3], and is expressed as:

$P\left(D_{0} \rightarrow \hat{D}_{0}\right) \leq \frac{1}{(\Gamma(\tilde{m}))^{N_{t} N_{r}}}\left[\prod_{i=0}^{N_{t}-1}\left(1+\frac{\rho}{4 N_{t}} \frac{\Omega_{0}}{\tilde{m}} \lambda_{i}\right)\right]^{-\tilde{m} N_{r}}$

where $\Gamma(\cdot)$ stands for Gamma function and $\left\{\lambda_{i}\right\}_{i=0}^{N_{t}-1}$ are the eigenvalues of the matrix $\mathbf{Z} \triangleq \boldsymbol{\Delta}_{D_{0}}^{T} \boldsymbol{\Delta}_{D_{0}}$. For single user system, since there is no effect of MA interference, $\rho$ reduces to $E_{0} / N_{0}$. As a result, the performance merits of UWB ST systems can be quantified similar to the narrowband case. That is with $r$ denoting the rank of $\mathbf{Z}, r \tilde{m} N_{r}$ is the diversity gain, and $(\Gamma(\tilde{m}))^{N_{t} /(\tilde{m} r)}\left(\Omega_{0} / \tilde{m}\right)\left(\prod_{i=0}^{r-1} \lambda_{i}\right)^{1 / r}$ is the coding gain.

\section{UWB ST CODES USING ROD}

\section{A. UWB ST Signals}

Considering a MIMO system with 2 transmit antennas, the THMPPM ST signal based on ROD [4] is given by:

$$
\tilde{\mathbf{X}}(t)=\sqrt{\frac{E}{N_{t}}} \sum_{m=0}^{M-1}\left(\begin{array}{cc}
s_{d, m}^{0} \tilde{w}_{m}^{0}(t) & s_{d, m}^{1} \tilde{w}_{m}^{0}(t) \\
-s_{d, m}^{1} \tilde{w}_{m}^{1}(t) & s_{d, m}^{0} \tilde{w}_{m}^{1}(t)
\end{array}\right),
$$

where the user subscript $u$ is omitted for notation simplicity. Since 2 frames are used to transmit 2 symbols, $d^{0}$ and $d^{1}$, the code is of full rate $R=1$. The $\mathrm{ST}$ signal constructed from ROD code with rate $R=1 / K$, where $K \geq 2$ is an even integer, can be modelled by a $K \times 2$ matrix [5]:

$$
\tilde{\mathbf{X}}(t)=\sqrt{\frac{E}{N_{t}}} \sum_{m=0}^{M-1} s_{d, m}\left(\begin{array}{cc}
\tilde{w}_{m}^{0}(t) & \tilde{w}_{m}^{0}(t) \\
-\tilde{w}_{m}^{1}(t) & \tilde{w}_{m}^{1}(t) \\
\vdots & \vdots \\
\tilde{w}_{m}^{K-2}(t) & \tilde{w}_{m}^{K-2}(t) \\
-\tilde{w}_{m}^{K-1}(t) & \tilde{w}_{m}^{K-1}(t)
\end{array}\right)_{K \times 2}
$$

In this case, the data symbol $d$ is transmitted repeatedly over $K$ frames from both transmit antennas. ROD ST signal utilizing BPSK technique can be defined similar to that of TH-MPPM system by replacing $\sum_{m=0}^{M-1} s_{d, m}^{i} \tilde{w}_{m}^{k}(t)$ with $d^{i} \tilde{w}\left(t-k T_{f}-c_{u}(k) T_{c}\right)$ and $d^{i} \sqrt{1 / N_{c}} \sum_{l=0}^{N_{c}-1} c_{u}(l) \tilde{w}\left(t-k T_{f}-l T_{c}\right)$ for TH and DS BPSK schemes, respectively.

\section{B. PEP Evaluation}

In this section, we calculate the PEP of UWB systems employing ROD ST codes with full and reduced rate. For TH-MPPM system, the $2 M \times 2$ matrix $\mathbf{S}_{D}$ corresponding to the transmitted ST signal given in (22) can be expressed as:

$$
\mathbf{S}_{D}=\left(\begin{array}{cc}
\mathbf{s}_{d}^{0}(0) & \mathbf{s}_{d}^{1}(0) \\
\mathbf{s}_{d}^{0}(1) & \mathbf{s}_{d}^{1}(1)
\end{array}\right)=\left(\begin{array}{cc}
\mathbf{s}_{d}^{0} & \mathbf{s}_{d}^{1} \\
-\mathbf{s}_{d}^{1} & \mathbf{s}_{d}^{0}
\end{array}\right)
$$

where $\mathbf{s}_{d}^{i}$ is a unit vector of length $M$ in which only the $\left(d^{i}\right)^{t h}$ entry is nonzero. Next, we can show that $\mathbf{Z}=2 \sum_{i=0}^{1} \delta\left(d^{i}-\hat{d}^{i}\right) \mathbf{I}_{2}$, where $\mathbf{I}_{N}$ is $N \times N$ identity matrix. Here, the two eigenvalues of $\mathbf{Z}$ are equal to $2 \sum_{i=0}^{1} \delta\left(d^{i}-\hat{d}^{i}\right)$. Assuming that $D$ and $\hat{D}$ are two distinct codewords, $\mathbf{Z}$ is of full rank $r=2$. In this case, UWB ROD ST code offers full diversity of $2 N_{r}$. For ROD ST code with rate $1 / K$, we have

$$
\mathbf{S}_{D}=\left(\left(\begin{array}{cc}
\mathbf{s}_{d} & \mathbf{s}_{d} \\
-\mathbf{s}_{d} & \mathbf{s}_{d}
\end{array}\right)^{T} \cdots\left(\begin{array}{cc}
\mathbf{s}_{d} & \mathbf{s}_{d} \\
-\mathbf{s}_{d} & \mathbf{s}_{d}
\end{array}\right)^{T}\right)_{K \times 2}^{T}
$$

It can be shown that the matrix $\mathbf{Z}=2 K \delta(d-\hat{d}) \mathbf{I}_{2}$ is also of full rank with two equal eigenvalues $\lambda_{i}=2 K \delta(d-\hat{d})$. Similarly, employing BPSK system, we can show that $\mathbf{Z}=\lambda \mathbf{I}_{2}$, where $\lambda=4 \sum_{i=0}^{1} \delta\left(d^{i}-\hat{d}^{i}\right)$ for full rate and $\lambda=4 K \delta(d-\hat{d})$ for $1 / K$ rate are the eigenvalues of $\mathbf{Z}$. Note that with $d \neq \hat{d}$, the matrix $\mathbf{Z}$ in both cases is of full rank, and hence the maximum diversity gain can be achieved.

Substituting the nonzero eigenvalues of $\mathbf{Z}, \lambda_{0}=\lambda_{1} \triangleq \lambda$ into (21), the PEP upper bound can be simplified to:

$$
P\left(D_{0} \rightarrow \hat{D}_{0}\right) \leq(\Gamma(\tilde{m}))^{-2 N_{r}}\left(1+\frac{\rho \lambda}{8} \frac{\Omega_{0}}{\tilde{m}}\right)^{-2 \tilde{m} N_{r}} .
$$

We can observe from (23) that for fixed $N_{r}, \tilde{m}$, and $\Omega_{0}$, the average PEP depends on the value of $\rho \lambda$. The higher the $\rho \lambda$, the better the performance. To compare the performance of various systems, we assume that the energy per bit $E_{b}$ is fixed. Expressing $E$ in term of $E_{b}$, we have $E=E_{b} \log _{2}(M)$ for full rate and $E=$ $E_{b} \log _{2}(M) / K$ for $1 / K$ rate ROD ST codes. Considering single user system employing a particular modulation, we observe that 
with only one erroneous symbol, both full and reduced rate ROD ST codes result in the same value of $\rho \lambda$, and hence yield similar performance. On the other hand, for multiuser case, consider $\rho$ as computed in (20) and denote the eigenvalue of full rate ROD ST system as $\bar{\lambda}$. Assuming $E_{0}=\cdots=E_{N_{u}-1} \triangleq E$ and $\Omega_{0}=$ $\cdots=\Omega_{N_{u}-1} \triangleq \Omega$, we obtain

$$
\rho \lambda=\left[2 \sigma_{a}^{2} \Omega\left(N_{u}-1\right)+\left(E_{b} \log _{2}(M) / N_{0}\right)^{-1}\right]^{-1} \bar{\lambda},
$$

for full rate, and

$$
\rho \lambda=\left[2 \sigma_{a}^{2} \Omega\left(N_{u}-1\right) / K+\left(E_{b} \log _{2}(M) / N_{0}\right)^{-1}\right]^{-1} \bar{\lambda}
$$

for ROD ST codes with $1 / K$ rate. Since $\rho \lambda$ in case of full rate is less than that of reduced rate, reducing the code rate is likely to improve the performance of multiuser system. To compare the performances of various modulation systems, we observe that with a fixed code rate, the eigenvalues of TH-BPSK and DSBPSK systems are twice as those of TH-BPPM system. Therefore, both TH-BPSK and DS-BPSK systems tend to outperform THBPPM system for every code rate.

\section{UWB-MIMO PERFormanCE IN MUltipath CHANNElS}

Taking into account the frequency-selectivity, a more realistic channel for UWB system is represented by a stochastic tappeddelayed-line model, $\sum_{l=0}^{L_{u}-1} h_{u}^{i j}(l) \delta\left(t-\tau_{u}(l)\right)$, where $\left\{h_{u}^{i j}(l)\right\}$ denote the multipath gain coefficients, $\left\{\tau_{u}(l)\right\}$ are the corresponding path delays, and $\left\{L_{u}\right\}$ are the number of resolvable paths. Based on this channel model, the received signal in (5) becomes

$$
r^{j}(t)=\sum_{u=0}^{N_{u}-1} \sum_{i=0}^{N_{t}-1} \sum_{l=0}^{L_{u}-1} h_{u}^{i j}(l) x_{u}^{i}\left(t-\tau_{u}(l)\right)+n^{j}(t) .
$$

In order to capture the energy carried by multipath signals, $L$ finger $\left(L \leq L_{u}\right)$ Rake receiver is used at each receive antenna. Assuming independent multipath channels, we have shown in [12] that the diversity gain can increase up to $r \tilde{m} N_{r} L$. For example, for TH-BPSK, the PEP is upper bounded by:

$P\left(D_{0} \rightarrow \hat{D}_{0}\right) \leq f(\tilde{m})\left[\prod_{l=0}^{L-1} \prod_{i=0}^{N_{t}-1}\left(1+\frac{\rho}{4 N_{t}} \frac{\Omega_{0}(l)}{\tilde{m}} \lambda_{i}\right)\right]^{-\tilde{m} N_{r}}$,

where $f(\tilde{m})=(\Gamma(\tilde{m}))^{-N_{t} N_{r} L}$ and $\rho=\frac{E_{0}}{2 \sigma_{n}^{2}}$. The analysis for both TH and DS systems is presented in details in [12].

\section{Simulation Results}

To support the analytical results in the previous sections, we performed simulations for UWB MA systems based on THMPPM, TH-BPSK and DS-BPSK schemes. We employ UWB signals with frame interval $T_{f}=100 \mathrm{~ns}$ and pulse duration $T_{w}$ of 0.7 ns. The received monocycle is modelled as the second derivative of the Gaussian pulse [9]:

$$
w(t)=\sqrt{(8 / 3)}(1-4 \alpha) \exp (-2 \alpha)
$$

where $\alpha=\pi\left(t / \tau_{o}\right)^{2}$ and $\tau_{o}$ parameterizes the width of the monocycle. Here, we select $\tau_{o}=0.2877 \mathrm{~ns}$ in order to yield the pulse width of $0.7 n s$, as in [9]. The factor $\sqrt{8 / 3}$ is introduced such that each pulse has unit energy. The autocorrelation function of the pulse in (26) is given by [9]:

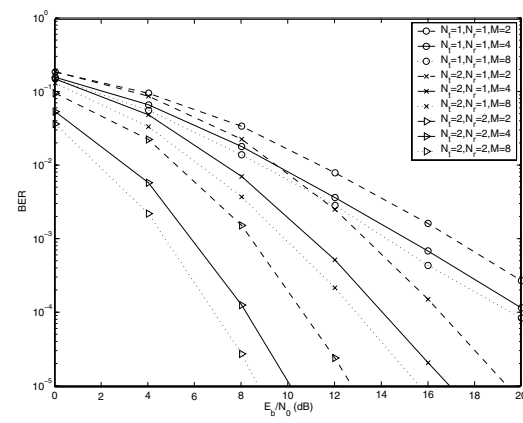

Fig. 3: TH-MPPM single user systems.

$$
\gamma(t)=\left(1-4 \alpha+(4 / 3) \alpha^{2}\right) \exp (-\alpha)
$$

where $\gamma(t)$ can be approximately zero when $|t| \geq T_{w}$. In THMPPM system, the modulation delays $\left\{T_{m}\right\}_{m=0}^{M-1}$ are selected to be $T_{m}=m T_{w}$, i.e., the two consecutive $M$-ary symbols have different time shift of $T_{w}$ seconds. The TH is therefore $T_{c}=M T_{w}$ seconds. For TH-BPSK and DS-BPSK systems whose $d_{u}^{i}(k) \in\{-1,1\}, T_{c}$ can be chosen to be equal to the pulse width $\left(T_{c}=T_{w}\right)$ since BPSK scheme does not require any additional time delay for data modulation. To avoid cross-frame collisions in MA system, total hop interval is limited to $T_{f} / 2$. Here, we adopt discrete uniform random $\mathrm{TH}$ and spreading sequence so as to evaluate the performances regardless of the choice of any particular code, as in [7], [8].

In this section, we show simulation results for the performance of UWB ROD ST block codes with different rates for real Nakagami-2 flat fading channels over $K$ symbol periods. Each channel coefficient is normalized to have unit power, i.e., $\Omega=1$. The thermal noise is real Gaussian random process with zero mean and variance $N_{0} / 2$. The channel coefficients, transmitted signals, and noise are generated independently. Fig. 3 shows the BER performance of TH-MPPM single user system versus $E_{b} / N_{0}$ for modulation sizes $M=2,4$, and 8 . We can see that the MIMO systems outperform the SISO systems for every modulation size. In addition, we observe that the performance improvement obtained from ROD ST code is considerably higher in high Signal-to-Noise Ratio (SNR) regime.

In Fig. 4, we illustrate the performances of single user systems employing binary data transmission $(M=2)$ with different modulation and MA techniques. Similar to the previous observation, increasing the number of antennas improves the BER performance of every modulation system. In addition, we can see that BPSK systems yield better performance than BPPM systems. This is due to the fact that for equal transmitted energy, BPSK which is antipodal signalling has greater distance than BPPM which is an orthogonal signalling scheme. This observation supports our theoretical analysis in (23) in the previous section.

Next, we consider multiuser system with various modulations. We assume that all users transmit with equal power. Fig. 5 presents the performances of MA systems with $N_{u}=10$. Again, the MIMO systems perform better than the SISO systems for any number of active users. Results also show that the performances of both SISO and MIMO systems degrade when more users are presented. In contrast to the single user case, by employing $\mathrm{TH}$ techniques, increasing SNR slightly improves the performance of 


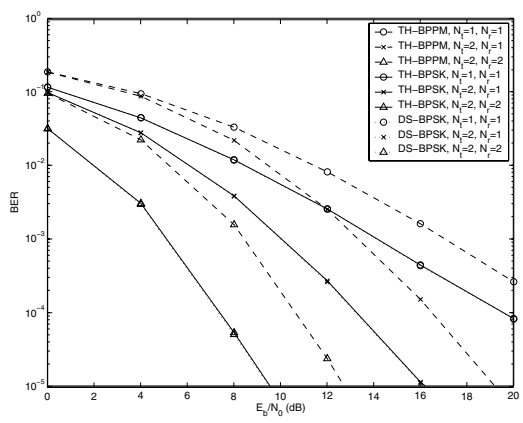

Fig. 4: TH and DS UWB single user systems.

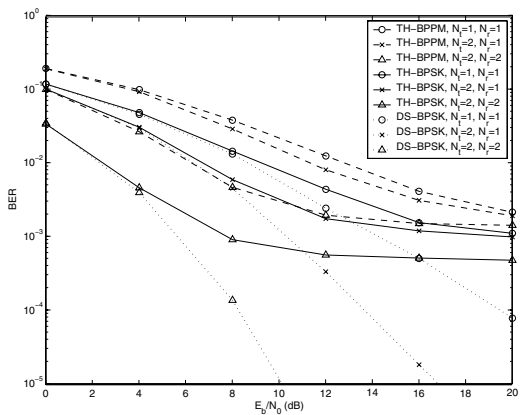

Fig. 5: TH and DS UWB multiuser systems $\left(N_{u}=10\right)$.

the multiuser systems. This is due to the fact that in high SNR regime, it is the effect of multiuser that prevails, regardless of the $E_{b} / N_{0}$. However, since the MA interference is considerably small when DS technique is utilized, DS ST systems yield remarkable performance improvement even in MA scenarios.

In Fig. 6 and 7, we demonstrate the performances of UWB ROD ST codes with full and half rates for single and multiple user $\left(N_{u}=10\right)$ systems, respectively. We see from Fig. 6 that the performances of full and half rate ROD codes are close to each other. This observation is in agreement with (23) in Section V that for single user system, decreasing the rate of ROD ST code does not improve the performance. On the contrary, Fig. 7 shows that with $N_{u}=10$, reducing the code rate improves the performances of TH ST systems. However, utilizing ROD code with either full or reduced rate yields similar performances for DS systems due to the minimal effect of MA interference. Comparing all of the schemes, we can conclude that DS ST systems provide the best performance in MA environment.

\section{CONCLUSIONS}

In this paper, we investigated the UWB ST systems utilizing TH-MPPM, TH-BPSK, and DS-BPSK signals. The performance metrics (diversity and coding gains) of UWB ST systems were quantified for every modulation technique regardless of the particular coding scheme. We considered an example of UWB ST signals based on ROD ST code for 2 transmit antenna system. Both analytical and simulation results showed the performance improvement of the UWB MIMO systems over the conventional SISO systems. By employing 2 transmit and 2 receive antennas for a system of 10 users and $E_{b} / N_{0}=8 \mathrm{~dB}$, the BER improvement for TH-BPPM was from $3.8 \times 10^{-2}$ to $4.6 \times 10^{-3}$, for TH-BPSK was from $1.4 \times 10^{-2}$ to $9 \times 10^{-4}$, and for DS-BPSK

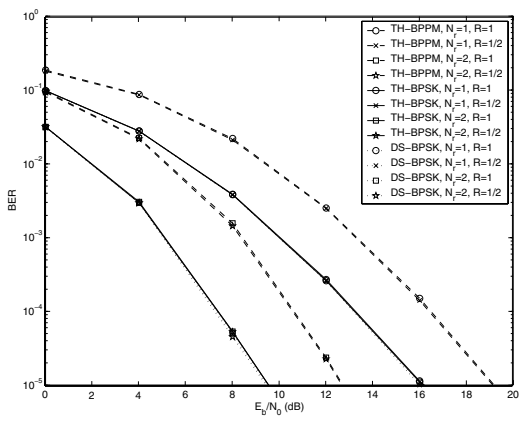

Fig. 6: UWB single user systems with ROD ST codes of different rates.

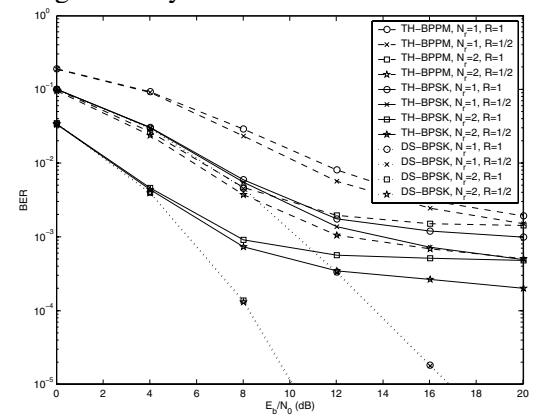

Fig. 7: UWB multiuser systems with ROD ST codes of different rates.

was from $1.3 \times 10^{-2}$ to $1.3 \times 10^{-4}$. We illustrated that in single user case, both DS-BPSK and TH-BPSK yielded similar performance which was superior to TH-BPPM system, whereas in MA scenarios, DS-BPSK outperformed other considered schemes.

\section{REFERENCES}

[1] M. L. Welborn, "System Considerations for Ultra-Wideband Wireless Networks", IEEE Radio and Wireless Conf., pp. 5-8, Aug. 2001.

[2] M. Z. Win and R. A. Scholtz, "Impulse Radio: How It Works", IEEE Commun. Letters, vol. 2, no. 2, pp. 36-38, Feb. 1998.

[3] V. Tarokh, N. Seshadri and A. R. Calderbank, "Space-Time Codes for High Data Rate Wireless Communication: Performance Criterion and Code Construction", IEEE Trans. on Inform. Theory, vol. 44, no. 2, pp. 744-765, Mar. 1998.

[4] V. Tarokh, H. Jafarkhani and A. R. Calderbank, "Space-Time Block Codes from Orthogonal Designs", IEEE Trans. on Inform. Theory, vol. 45, no. 5, pp. 1456-1467, Jul. 1999.

[5] L. Yang and G. B. Giannakis, "Space-Time Coding for Impulse Radio", IEEE Conf. on Ultra Wideband Systems and Technologies, pp. 235-240, May 2002

[6] J. R. Foerster, "The Performance of a Direct-Sequence Spread UltraWideband System in the Presence of Multipath, Narrowband Interference, and Multiuser Interference ", IEEE Conf. on Ultra Wideband Systems and Technologies, pp. 87-91, May 2002.

[7] V. S. Somayazulu, "Multiple Access Performance in UWB Systems Using Time Hopping vs. Direct Sequence Spreading", IEEE Wireless Commu. and Networking Conf., vol. 2, pp. 522-525, Mar. 2002.

[8] G. Durisi and S. Benedetto, "Performance Evaluation and Comparison of Different Modulation Schemes for UWB Multiaccess Systems", IEEE Int. Conf. on Commun., vol. 3, pp. 2187-2191, May 2003.

[9] M. Z. Win and R. A. Scholtz, "Ultra-Wide Bandwidth Time-Hopping Spread-Spectrum Impulse Radio for Wireless Multiple-Access Communications", IEEE Trans. on Commun., vol. 48, no. 4, pp. 679-691, Apr. 2000.

[10] C. J. Le Martet and G. B. Giannakis, "All-digital PPM Impulse Radio for Multiple-Access through Frequency-Selective Multipath", Proc. IEEE Sensor Array and Multichannel Sig. Proc. Workshop, pp. 22-26, Mar. 2000.

[11] J. G. Proakis, Digital Communications, McGraw-Hill, New York, 2001

[12] W. Siriwongpairat, M. Olfat, and K. J. R. Liu, "Performance Analysis and Comparison of TH and DS UWB Systems employing MIMO Transmission", EURASIP J. on Applied Signal Processing Special Issue on "UWB-State of the Art", submitted Oct. 2003, under revision. 